\title{
Quantifying the biodistribution of nanoparticles
}

To the Editor - Yamashita et al. (Nature Nanotech. 6, 321-328; 2011) report that silica and titanium dioxide $\left(\mathrm{TiO}_{2}\right)$ nanoparticles with diameters of $70 \mathrm{~nm}$ and $35 \mathrm{~nm}$, respectively, can cross the placental barrier in pregnant mice. Using transmission electron microscopy (TEM), the researchers claim that nanoparticles are found in the liver and brain of the fetus ${ }^{1}$. Although TEM is useful for the qualitative examination of nanoparticles, it is not sensitive enough for studying the trans-placental transport of $\mathrm{TiO}_{2}$ nanoparticles.

Assuming that the concentration of $\mathrm{TiO}_{2}$ nanoparticles in the fetal liver is one nanogram per gram of liver (the density of liver is approximately $1.1 \mathrm{~g} \mathrm{~cm}^{-3}$ ) and that the mass of a $35 \mathrm{~nm} \mathrm{TiO}_{2}$ particle is approximately $1 \times 10^{-16} \mathrm{~g}$ (the density of rutile- $\mathrm{TiO}_{2}$ is $4.3 \mathrm{~g} \mathrm{~cm}^{-3}$ ), on average, only one nanoparticle can theoretically be found in a $1 \mathrm{~mm}^{2}$ section of liver tissue (an ultrathin section usually has a thickness of less than $100 \mathrm{~nm}$ ). The TEM images collected by Yamashita et al. showed a dark electron-dense spot in a field size of $\sim 5 \mu \mathrm{m} \times 5 \mu \mathrm{m}$. Based on our estimation, on average, tens of thousands of such images need to be examined to find one $\mathrm{TiO}_{2}$ nanoparticle. This means that the TEM results cannot firmly prove that nanoparticles were present in the fetal liver and brain, unless the concentration of nanoparticles in the fetal liver is several orders of magnitude higher than the hypothetical value of one nanogram per gram.
In conclusion, more suitable quantitative methods should be used to study the biodistribution of nanoparticles in pregnant mice.

Xiao He1, Zhiyong Zhang'* , Jinsen Liu², YuhuiMa', Peng Zhang', Yuanyuan $\mathrm{Li}^{1}$, Zhenqiang $\mathrm{Wu}^{2}$, Yuliang Zhao ${ }^{1 *}$ and Zhifang Chai ${ }^{1}$

${ }^{1}$ CAS Key Laboratory for Biomedical Effects of Nanomaterials and Nanosafety, and Key Laboratory of Nuclear Analytical Techniques, Institute of High Energy Physics, Chinese Academy of Sciences, Beijing 100049, China. ${ }^{2} \mathrm{~S} c h o o l$ of Biological Science and Engineering, South China University of Technology,

Guangzhou 510641 China.

*e-mail: zhangzhy@ihep.ac.cn; zhaoyuliang@ihep.ac.cn
To the Editor - In general, transmission electron microscopy (TEM) and quantitative methods such as inductively coupled plasma-mass spectrometry (ICP-MS) are used to study the biodistribution of nanomaterials. For example, ICP-MS can detect the elements of nanomaterials and evaluate their biodistribution quantitatively. However, ICP-MS cannot distinguish between elements that are inherent to the nanomaterials and those that are cleaved or released from them. But, unlike ICP-MS, TEM can detect the presence of nanomaterials and identify their location within tissues and cells. Even though the TEM images in our study ${ }^{1}$ provide only qualitative information, TEM is invaluable for identifying the biodistribution of the silica and $\mathrm{TiO}_{2}$ nanoparticles.

He et al. ${ }^{2}$ correctly point out the detection limit of TEM and that only some silica and $\mathrm{TiO}_{2}$ nanoparticles could be detected in the small section of the placental and fetal tissue in our study. However, through analysis of several hundreds of TEM sections, we confirmed that silica and $\mathrm{TiO}_{2}$ nanoparticles did accumulate in both the placental and fetal tissues. The observations were not coincidental.

To study the biodistribution of nanomaterials quantitatively, methods such as ICP-MS should be used and, indeed quantitative biodistribution studies of silica and $\mathrm{TiO}_{2}$ nanoparticles in the mouse placenta and fetus are currently under way in our laboratory.

References

1. K. Yamashita, et al. Nature Nanotech. 6, 321-328 (2011)

2. He et al. Nature Nanotech. 6, 755 (2011).

Yasuo Tsutsumi ${ }^{\star}$ and Yasuo Yoshioka on behalf of all the authors of ref. 1

Department of Toxicology and Safety Science, Osaka University, 1-6 Yamadaoka, Suita, Osaka 565-0871, Japan. National Institute of Biomedical Innovation, 7-6-8, Saito-Asagi, Ibaraki, Osaka 567-0085, Japan. The Center for Advanced Medical Engineering and Informatics, Osaka University, 1-6 Yamadaoka, Suita, Osaka 565-0871, Japan.

*e-mail: ytsutsumi@phs.osaka-u.ac.jp 